\title{
Frame properties of systems arising via iterated actions of operators
}

\author{
Christensen, Ole; Hasannasabjaldehbakhani, Marzieh
}

Published in:

Applied and Computational Harmonic Analysis

Link to article, DOI:

10.1016/j.acha.2018.04.002

Publication date:

2018

Document Version

Peer reviewed version

Link back to DTU Orbit

Citation (APA):

Christensen, O., \& Hasannasabjaldehbakhani, M. (2018). Frame properties of systems arising via iterated actions of operators. Applied and Computational Harmonic Analysis, 46(3), 664-673.

https://doi.org/10.1016/j.acha.2018.04.002

\section{General rights}

Copyright and moral rights for the publications made accessible in the public portal are retained by the authors and/or other copyright owners and it is a condition of accessing publications that users recognise and abide by the legal requirements associated with these rights.

- Users may download and print one copy of any publication from the public portal for the purpose of private study or research.

- You may not further distribute the material or use it for any profit-making activity or commercial gain

- You may freely distribute the URL identifying the publication in the public portal

If you believe that this document breaches copyright please contact us providing details, and we will remove access to the work immediately and investigate your claim. 


\section{Accepted Manuscript}

Frame properties of systems arising via iterated actions of operators

Ole Christensen, Marzieh Hasannasab

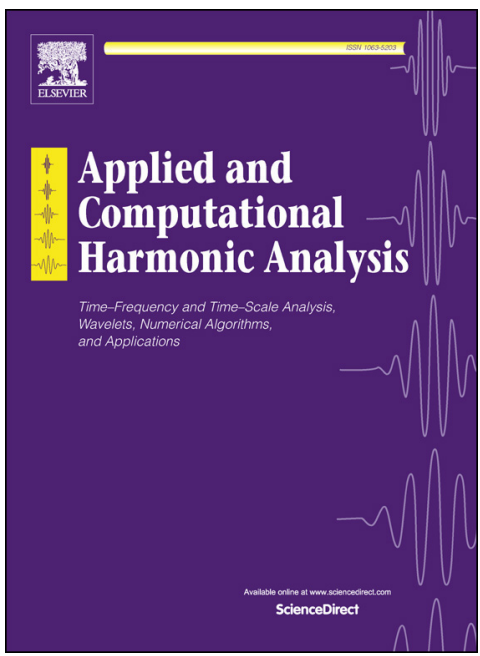

To appear in: $\quad$ Applied and Computational Harmonic Analysis

Received date: 29 June 2016

Revised date: 31 January 2018

Accepted date: 14 April 2018

Please cite this article in press as: O. Christensen, M. Hasannasab, Frame properties of systems arising via iterated actions of operators, Appl. Comput. Harmon. Anal. (2018), https://doi.org/10.1016/j.acha.2018.04.002

This is a PDF file of an unedited manuscript that has been accepted for publication. As a service to our customers we are providing this early version of the manuscript. The manuscript will undergo copyediting, typesetting, and review of the resulting proof before it is published in its final form. Please note that during the production process errors may be discovered which could affect the content, and all legal disclaimers that apply to the journal pertain. 


\title{
Frame properties of systems arising via iterated actions of operators
}

\author{
Ole Christensen, Marzieh Hasannasab
}

April 17, 2018

\begin{abstract}
Motivated by recent progress in dynamical sampling we prove that every frame which is norm-bounded below can be represented as a finite union of sequences $\left\{\left(T_{j}\right)^{n} \varphi_{j}\right\}_{n=0}^{\infty}, j=1, \ldots, J$ for some bounded operators $T_{j}$ and elements $\varphi_{j}$ in the underlying Hilbert space. The result is optimal, in the sense that it turns out to be problematic to replace the collection of generators $\varphi_{1}, \ldots, \varphi_{J}$ by a singleton: indeed, for linearly independent frames we prove that we can represent the frame in terms of just one system $\left\{T^{n} \varphi\right\}_{n=0}^{\infty}$, but unfortunately this representation often forces the operator $T$ to be unbounded. Several examples illustrate the connection of the results to typical frames like Gabor frames and wavelet frames, as well as generic constructions in arbitrary separable Hilbert spaces.
\end{abstract}

\section{Introduction}

The topic of dynamical sampling has recently attracted considerably attention, see, e.g., the papers $[2,1,3,19]$. The topic is motivated from concrete applications; from the pure mathematical point of view one of the key issues is to analyse frame properties for families of vectors arising by iterated actions of a class of operators on a family of generators. Thus, the main ingredients are a Hilbert space $\mathcal{H}$, and a class of operators $T_{j}: \mathcal{H} \rightarrow \mathcal{H}$ and corresponding vectors $\varphi_{j} \in \mathcal{H}$, for $j$ belonging to an index set $I$. Typically the Hilbert space is infinite-dimensional and the index set $I$ is finite.

Several of the results in the mentioned papers deal with properties of the resulting system

$$
\bigcup_{j \in I}\left\{T_{j}^{n} \varphi_{j}\right\}_{n=0}^{\infty}
$$

under various assumptions on the operators $T_{j}$. In this paper we will look at the issue from another angle. Indeed, we will take a given frame $\left\{f_{k}\right\}_{k=1}^{\infty}$ as 
the starting point, and ask for conditions such that $\left\{f_{k}\right\}_{k=1}^{\infty}$ can be decomposed into the form (1.1) with a finite index set $I$. We will show that such a decomposition is possible with certain bounded operators $T_{j}$ if $\left\{f_{k}\right\}_{k=1}^{\infty}$ is norm-bounded below; this makes the result applicable to most of the standard frames in applied harmonic analysis, e.g., Gabor frames, wavelet frames, generalized shift-invariant systems, etc. Technically our main tool is the Feichtinger Conjecture, which has been confirmed in [18] and therefore now is a theorem.

It is natural to ask for conditions under which a decomposition of the form (1.1) is possible for a singleton $I$, i.e., with just a single generator $\varphi$; or, if multi-generators $\varphi_{1}, \ldots, \varphi_{J}$ are necessary, whether all the operators $T_{j}$ can be chosen to be identical. Both issues turn out to be problematic: while we indeed can give a simple characterization of the case where a frame can be written in the form $\left\{T^{n} \varphi\right\}_{n=0}^{\infty}$ for some $\varphi \in \mathcal{H}$ and an operator $T$, this representation might force $T$ to be unbounded even for very simple overcomplete frames. The same remark applies to the multi-generator case. This result justifies some of the no-go results in the papers [2, 1, 3], typically of the form that certain conditions on the involved operators (including boundedness) would imply that the iterated system can not be a frame. Other papers dealing with iterated actions of operators include $[11,12]$.

Along the way we will relate the results to the frames appearing in applied harmonic analysis. For example, the results imply that any Gabor frame $\left\{E_{m b} T_{n a} g\right\}_{m, n \in \mathbb{Z}}$ (see the definition below) in $L^{2}(\mathbb{R})$ indeed has the form $\left\{T^{n} \varphi\right\}_{n=0}^{\infty}$ for some $\varphi \in L^{2}(\mathbb{R})$ and a certain operator $T: L^{2}(\mathbb{R}) \rightarrow L^{2}(\mathbb{R})$; if a representation using bounded operators is desired, we can obtain this as in (1.1) using multi-generators.

The paper is organized as follows. We will collect the main results and key examples in Section 2, and Section 3 is devoted to the technical proof of how to obtain the decomposition (1.1) for a frame that is norm-bounded below. In the rest of this section we will collect the necessary background from frame theory and motivate the problem via introductory examples and results. In the entire paper we let $\mathcal{H}$ denote an infinite-dimensional and separable Hilbert space.

A sequence $\left\{f_{k}\right\}_{k=1}^{\infty}$ in $\mathcal{H}$ is a frame for $\mathcal{H}$ if there exist constants $A, B>0$ such that

$$
A\|f\|^{2} \leq \sum_{k=1}^{\infty}\left|\left\langle f, f_{k}\right\rangle\right|^{2} \leq B\|f\|^{2}, \forall f \in \mathcal{H} .
$$

Frames are important because they lead to expansions of the elements in the underlying Hilbert space, similarly to what is known for orthonormal bases. Indeed, if $\left\{f_{k}\right\}_{k=1}^{\infty}$ is a frame for $\mathcal{H}$, the frame operator $S: \mathcal{H} \rightarrow \mathcal{H}, S f:=$ $\sum_{k=1}^{\infty}\left\langle f, f_{k}\right\rangle f_{k}$ is bounded and invertible, and each $f \in \mathcal{H}$ has the uncondi- 
tionally convergent expansion

$$
f=\sum_{k=1}^{\infty}\left\langle f, S^{-1} f_{k}\right\rangle f_{k} .
$$

The sequence $\left\{f_{k}\right\}_{k=1}^{\infty}$ is a frame sequence if the inequalities (1.2) hold for all $f \in \overline{\operatorname{span}}\left\{f_{k}\right\}_{k=1}^{\infty}$. The sequence $\left\{f_{k}\right\}_{k=1}^{\infty}$ is a Bessel sequence if at least the upper condition in (1.2) holds. Also, $\left\{f_{k}\right\}_{k=1}^{\infty}$ is called a Riesz sequence if there exist constants $A, B>0$ such that $A \sum\left|c_{k}\right|^{2} \leq\left\|\sum c_{k} f_{k}\right\|^{2} \leq B \sum\left|c_{k}\right|^{2}$ for all finite scalar sequences $c_{1}, \ldots, c_{N}, N \in \mathbb{N}$. A Riesz sequence is a Riesz basis if $\overline{\operatorname{span}}\left\{f_{k}\right\}_{k=1}^{\infty}=\mathcal{H}$; furthermore, the class of Riesz bases is precisely the class of frames $\left\{f_{k}\right\}_{k=1}^{\infty}$ for which the equation $\sum_{k=1}^{\infty} c_{k} f_{k}=0,\left\{c_{k}\right\}_{k=1}^{\infty} \in \ell^{2}(\mathbb{N})$, forces that $c_{k}=0$ for all $k \in \mathbb{N}$. Frequently the latter condition is expressed by saying that $\left\{f_{k}\right\}_{k=1}^{\infty}$ is $\omega$-independent. This is a much stronger condition than $\left\{f_{k}\right\}_{k=1}^{\infty}$ being linearly independent, which means that if a finite linear combination of vectors from $\left\{f_{k}\right\}_{k=1}^{\infty}$ is zero, all the coefficients must be zero.

A frame which is not a Riesz basis is said to be redundant or overcomplete. Certain frames (but not all) contain a Riesz basis as a subset. In this case the excess of the frame is defined as the number of elements that must be removed from the frame such that the remaining set is a Riesz basis. Note that the excess might be finite or infinite.

Gabor systems and wavelet systems are defined in terms of certain classes of operators on $L^{2}(\mathbb{R})$ : (i) translation by $a \in \mathbb{R}, T_{a} f(x)=f(x-a)$, (ii) modulation by $b \in \mathbb{R}, E_{b} f(x)=e^{2 \pi i b x} f(x)$, and (iii) dyadic scaling, $D f(x):=2^{1 / 2} f(2 x)$. In terms of these operators, a Gabor system on $L^{2}(\mathbb{R})$ has the form

$$
\left\{E_{m b} T_{n a} g\right\}_{m, n \in \mathbb{Z}}=\left\{e^{2 \pi i m b x} g(x-n a)\right\}_{m, n \in \mathbb{Z}},
$$

for some $a, b>0$ and a fixed function $g \in L^{2}(\mathbb{R})$. Similarly, the dyadic wavelet system generated by a function $\psi \in L^{2}(\mathbb{R})$ is the collection of functions

$$
\left\{D^{j} T_{k} \psi\right\}_{j, k \in \mathbb{Z}}=\left\{2^{j / 2} \psi\left(2^{j} x-k\right)\right\}_{j, k \in \mathbb{Z}}
$$

For Gabor systems as well as wavelet systems there is a huge literature on construction of frames and their properties. We refer to $[10,13,6]$ and the references therein.

As already mentioned, our goal is to consider representations of frames as finite unions of systems of vectors each arising via the iterated action of an operator on certain elements. In the simplest case of single-generated systems this means that we consider frames for $\mathcal{H}$ in the form $\left\{T^{n} \varphi\right\}_{n=0}^{\infty}$ for some linear operator $T$ and some $\varphi \in \mathcal{H}$. Let us first consider such systems for the translation operators on $\ell^{2}(\mathbb{Z})$ and $L^{2}(\mathbb{R})$, for this particular example with the index set $\{0\} \cup \mathbb{N}$ replaced by $\mathbb{Z}$ : 


\section{Example 1.1}

(i) Consider the Hilbert space $\ell^{2}(\mathbb{Z})$. The shift operator $T: \ell^{2}(\mathbb{Z}) \rightarrow \ell^{2}(\mathbb{Z})$, $T\left\{x_{k}\right\}_{k \in \mathbb{Z}}:=\left\{x_{k+1}\right\}_{k \in \mathbb{Z}}$, is linear, bounded, and invertible. Furthermore, letting $\delta=\left\{\delta_{k}\right\}_{k \in \mathbb{Z}} \in \ell^{2}(\mathbb{Z})$ denote the sequence for which $\delta_{0}=1$ and $\delta_{k}=0$ for $k \neq 0$, clearly $\left\{T^{n} \delta\right\}_{n \in \mathbb{Z}}$ is an orthonormal basis for $\ell^{2}(\mathbb{Z})$.

(ii) Now consider the Hilbert space $L^{2}(\mathbb{R})$. The analogue of the shift operator on $\ell^{2}(\mathbb{Z})$ is the translation operators $T_{a} f(x):=f(x-a), a \in \mathbb{R}$. However, it is known [7] that $\left\{T_{n a} \varphi\right\}_{n \in \mathbb{Z}}$ can not be a frame for $L^{2}(\mathbb{R})$, regardless of the choice of the parameter $a \in \mathbb{R}$ and the function $\varphi \in L^{2}(\mathbb{R})$. Formulated in the current setting, this means that the powers $\left\{T_{a}^{n} \varphi\right\}_{n \in \mathbb{Z}}$ can not form a frame for $L^{2}(\mathbb{R})$.

Note that the results in [7] even show that if we replace the single function $\varphi \in L^{2}(\mathbb{R})$ by a finite collection of functions $\left\{\varphi_{j}\right\}_{j=1}^{J}$, the set $\cup_{j=1}^{J}\left\{T_{a}^{n} \varphi_{j}\right\}_{n \in \mathbb{Z}}$ can not be a frame for $L^{2}(\mathbb{R})$. On the other hand, if we allow infinite index sets, such constructions exist: for example, the system $\cup_{j \in \mathbb{Z}}\left\{T_{1}^{n} E_{j} \chi_{[0,1]}\right\}_{n \in \mathbb{Z}}$ is an orthonormal basis for $L^{2}(\mathbb{R})$.

Despite the result in Example 1.1 it is indeed possible to construct an orthonormal basis $\left\{T^{n} \varphi\right\}_{n \in \mathbb{Z}}$ or $\left\{T^{n} \varphi\right\}_{n=0}^{\infty}$ for $L^{2}(\mathbb{R})$ for certain choices of the operator $T$ and $\varphi \in L^{2}(\mathbb{R})$. This follows from the following observation which in fact works in any separable Hilbert space. The result is a special case of Proposition 2.5.

Example 1.2 Let $\left\{e_{k}\right\}_{k=1}^{\infty}$ denote an orthonormal basis for $\mathcal{H}$. Define the operator $T$ by $T e_{k}:=e_{k+1}, k \in \mathbb{N}$. Then $\left\{e_{k}\right\}_{k=1}^{\infty}=\left\{T^{n} e_{1}\right\}_{n=0}^{\infty}$, and $T$ extends by linearity and continuity to a bounded operator on $\mathcal{H}$.

In the concrete case in the Hilbert space $L^{2}(\mathbb{R})$, we can thus obtain an orthonormal basis of the form $\left\{T^{n} e_{1}\right\}_{n=0}^{\infty}$ by taking any Gabor orthonormal basis $\left\{E_{m b} T_{n a} g\right\}_{m, n \in \mathbb{Z}}$ or wavelet orthonormal basis $\left\{D^{j} T_{k} \psi\right\}_{j, k \in \mathbb{Z}}$, reindex it as $\left\{e_{k}\right\}_{k=1}^{\infty}$, and apply the result directly.

\section{The results}

Our first result shows that any frame which is norm-bounded below is a finite union of iterated operator actions on certain elements:

Theorem 2.1 Consider a frame $\left\{f_{k}\right\}_{k=1}^{\infty}$ which is norm-bounded below. Then the following hold:

(i) The frame $\left\{f_{k}\right\}_{k=1}^{\infty}$ can be decomposed as a finite union

$$
\left\{f_{k}\right\}_{k=1}^{\infty}=\bigcup_{j=1}^{J}\left\{f_{k}^{(j)}\right\}_{k \in I_{j}},
$$


where each of the sequences $\left\{f_{k}^{(j)}\right\}_{k \in I_{j}}$ is an infinite Riesz sequence.

(ii) There is a finite collection of vectors from $\left\{f_{k}\right\}_{k=1}^{\infty}$, to be called $\varphi_{1}, \ldots, \varphi_{J}$, and corresponding bounded operators $T_{j}: \mathcal{H} \rightarrow \mathcal{H}$ with closed range, such that

$$
\left\{f_{k}\right\}_{k=1}^{\infty}=\bigcup_{j=1}^{J}\left\{T_{j}^{n} \varphi_{j}\right\}_{n=0}^{\infty}
$$

The proof of Theorem 2.1 needs some preparation, and will be given in Section 3. Since Gabor frames and wavelet frames (and any generalized shiftinvariant system) are norm-bounded below, the result applies directly to these cases. Note that the assumption of $\left\{f_{k}\right\}_{k=1}^{\infty}$ being norm-bounded below can not be removed in Theorem 2.1:

Example 2.2 Letting $\left\{e_{k}\right\}_{k=1}^{\infty}$ denote an orthonormal basis for $\mathcal{H}$, it is wellknown that the sequence $\left\{f_{k}\right\}_{k=1}^{\infty}=\left\{e_{1}, \frac{1}{\sqrt{2}} e_{2}, \frac{1}{\sqrt{2}} e_{2}, \frac{1}{\sqrt{3}} e_{3}, \frac{1}{\sqrt{3}} e_{3}, \frac{1}{\sqrt{3}} e_{3}, \ldots\right\}$ is a tight frame. The decomposition (2.1) in Theorem 2.1 is clearly impossible for this frame, showing that the assumption of $\left\{f_{k}\right\}_{k=1}^{\infty}$ being norm-bounded below can not be removed.

Theorem 2.1 leads to a couple of natural questions concerning possible simplifications of the structure of the decomposition (2.1). For example, what are the conditions such that a decomposition as in (2.1) is possible with $J=$ 1, i.e., a single generator? And if it is necessary to apply multi-generators $\varphi_{1}, \ldots, \varphi_{J}$, can the corresponding operators $T_{j}$ be chosen to be independent of $j$ ?

In order to answer these questions we will first provide a characterization of the frames $\left\{f_{k}\right\}_{k=1}^{\infty}$ that have the form $\left\{f_{k}\right\}_{k=1}^{\infty}=\left\{T^{n} \varphi\right\}_{n=0}^{\infty}$ for some linear operator $T$ on (a subspace of) $\mathcal{H}$ and some $\varphi \in \mathcal{H}$. In fact, we can state the result under a weaker assumption. Recall that when we consider $\left\{f_{k}\right\}_{k=1}^{\infty}$ as a sequence, we have determined a certain ordering on the elements. Due to the ordering, the condition $\left\{f_{k}\right\}_{k=1}^{\infty}=\left\{T^{n} \varphi\right\}_{n=0}^{\infty}$ actually implies that $\varphi=f_{1}$.

Proposition 2.3 Consider any sequence $\left\{f_{k}\right\}_{k=1}^{\infty}$ in $\mathcal{H}$ for which span $\left\{f_{k}\right\}_{k=1}^{\infty}$ is infinite-dimensional. Then the following are equivalent:

(i) $\left\{f_{k}\right\}_{k=1}^{\infty}$ is linearly independent.

(ii) There exists a linear operator $T: \operatorname{span}\left\{f_{k}\right\}_{k=1}^{\infty} \rightarrow \mathcal{H}$ such that $\left\{f_{k}\right\}_{k=1}^{\infty}=$ $\left\{T^{n} f_{1}\right\}_{n=0}^{\infty}$.

Proof. In order to prove (i) $\Rightarrow$ (ii), assume that $\left\{f_{k}\right\}_{k=1}^{\infty}$ is linearly independent. Let $T f_{k}:=f_{k+1}, k \in \mathbb{N}$, and extend $T$ to an operator on $\operatorname{span}\left\{f_{k}\right\}_{k=1}^{\infty}$ by 
linearity; then (ii) holds. In order to prove (ii) $\Rightarrow$ (i), assume on the contrary that (ii) holds and that $\left\{f_{k}\right\}_{k=1}^{\infty}$ is linearly dependent. Clearly we have that $f_{1} \neq 0$. The linear dependence of $\left\{f_{k}\right\}_{k=1}^{\infty}$ shows that for some $N>1$ and some coefficients $\left\{c_{k}\right\}_{k=1}^{N} \neq 0$ we have $\sum_{k=1}^{N} c_{k} f_{k}=0$. We can assume that $c_{N} \neq 0$, which implies that $f_{N}=\sum_{k=1}^{N-1} d_{k} f_{k}$ for some coefficients $d_{k}$. Now let $V:=\operatorname{span}\left\{f_{k}\right\}_{k=1}^{N-1}=\operatorname{span}\left\{T^{n} f_{1}\right\}_{n=0}^{N-2}$. Then $f_{N} \in \operatorname{span}\left\{f_{k}\right\}_{k=1}^{N-1}=V$. The space $V$ is invariant under $T$; indeed for any coefficients $a_{k} \in \mathbb{C}$,

$$
T \sum_{k=1}^{N-1} a_{k} f_{k}=\sum_{k=1}^{N-2} a_{k} f_{k+1}+a_{N-1} T f_{N-1}=\sum_{k=1}^{N-2} a_{k} f_{k+1}+a_{N-1} f_{N} \in V .
$$

Thus (ii) implies that $\operatorname{span}\left\{f_{k}\right\}_{k=1}^{\infty}=V$, which is a contradiction. We conclude that $\left\{f_{k}\right\}_{k=1}^{\infty}$ is linearly independent if (ii) holds.

Note that Proposition 2.3 implies that the question of the existence of an operator $T: \operatorname{span}\left\{f_{k}\right\}_{k=1}^{\infty} \rightarrow \mathcal{H}$ such that a given frame $\left\{f_{k}\right\}_{k=1}^{\infty}$ can be represented in the form $\left\{T^{n} \varphi\right\}_{n=0}^{\infty}$ for some $\varphi \in \mathcal{H}$ is independent of the ordering of the elements in $\left\{f_{k}\right\}_{k=1}^{\infty}$.

Proposition 2.3 has immediate consequences for Gabor systems in $L^{2}(\mathbb{R})$ :

Example 2.4 It was conjectured in [15] and finally proved in [17] that for any $a, b>0$ and any nonzero $g \in L^{2}(\mathbb{R})$ the Gabor system $\left\{E_{m b} T_{n a} g\right\}_{m, n \in \mathbb{Z}}$ is linearly independent. Proposition 2.3 therefore implies that there is a (possibly unbounded) operator $T$ such that $\left\{E_{m b} T_{n a} g\right\}_{m, n \in \mathbb{Z}}=\left\{T^{n} \varphi\right\}_{n=0}^{\infty}$ for some $\varphi \in$ $L^{2}(\mathbb{R})$; one choice could be $\varphi=g$.

Note that [15] even conjectured that any Gabor system $\left\{E_{b_{m}} T_{a_{m}} g\right\}_{m \in I}$ with $g \neq 0$ and distinct points $\left\{\left(a_{m}, b_{m}\right)\right\}_{m \in I}$ is linearly independent. Thus, if this is true, we would again obtain a representation $\left\{E_{b_{m}} T_{a_{m}} g\right\}_{m \in I}=\left\{T^{n} \varphi\right\}_{n=0}^{\infty}$ for some $\varphi \in L^{2}(\mathbb{R})$, under the assumption that $I$ is countable and infinite. The conjecture has been verified in several cases, but the full conjecture is still open. See [14] for a detailed discussion.

Note that in the case of a wavelet system, certain technical conditions for linear independence can be found in the papers [9] and [4].

In the rest of this section we will relate Proposition 2.3 and Theorem 2.1. First, in Theorem 2.1 we know that the operators $T_{j}$ can be chosen to be bounded. For Riesz sequences $\left\{f_{k}\right\}_{k=1}^{\infty}$ we now prove that also the operator $T$ in Proposition 2.3 can be chosen to be bounded, but we will soon see that this is not necessarily the case if $\left\{f_{k}\right\}_{k=1}^{\infty}$ is redundant. Thus, the reduction from multi-generators to a singleton is in general not desirable.

\section{Proposition 2.5}

(i) Any Riesz sequence $\left\{e_{k}\right\}_{k=1}^{\infty}$ in $\mathcal{H}$ has the form $\left\{e_{k}\right\}_{k=1}^{\infty}=\left\{T^{n} e_{1}\right\}_{n=0}^{\infty}$ for some operator $T \in B(\mathcal{H})$ with closed range. 
(ii) If $\left\{T^{n} \varphi\right\}_{n=0}^{\infty}$ is a frame for some operator $T \in B(\mathcal{H})$ and some $\varphi \in \mathcal{H}$, then $T$ has closed range.

Proof. Assume that $\left\{e_{k}\right\}_{k=1}^{\infty}$ is a Riesz sequence. By Lemma 3.6.5 in [6] applied on the Hilbert space $\overline{\operatorname{span}}\left\{e_{k}\right\}_{k=1}^{\infty}$, the mapping $T e_{k}:=e_{k+1}, k \in \mathbb{N}$, extends to a bounded linear operator $T: \overline{\operatorname{span}}\left\{e_{k}\right\}_{k=1}^{\infty} \rightarrow \mathcal{H}$. Clearly the range of $T$ is $\mathcal{R}_{T}=\overline{\operatorname{span}}\left\{e_{k}\right\}_{k=2}^{\infty}$, and by construction $\left\{e_{k}\right\}_{k=1}^{\infty}=\left\{T^{n} e_{1}\right\}_{n=0}^{\infty}$. This proves (i). In order to prove (ii), assume that $\left\{T^{n} \varphi\right\}_{n=0}^{\infty}$ is a frame; then $\left\{T^{n} \varphi\right\}_{n=1}^{\infty}$ is a frame sequence. Thus, the operator $U: \ell^{2}(\mathbb{N}) \rightarrow \mathcal{H}, U\left\{c_{k}\right\}_{k=1}^{\infty}=\sum_{k=1}^{\infty} c_{k} T^{k} \varphi$ has closed range, namely, $\overline{\operatorname{span}}\left\{T^{n} \varphi\right\}_{n=1}^{\infty}$. Now, for any $\left\{c_{k}\right\}_{k=1}^{\infty} \in \ell^{2}(\mathbb{N})$, we have $\sum_{k=1}^{\infty} c_{k} T^{k} \varphi=T \sum_{k=1}^{\infty} c_{k} T^{k-1} \varphi$; this implies that $\mathcal{R}_{T}=\overline{\operatorname{span}}\left\{T^{n} \varphi\right\}_{n=1}^{\infty}$.

Note that the result in Proposition 2.5 (ii) does not extend to multiple generators. Indeed, if $\cup_{j=1}^{J}\left\{T_{j}^{n} \varphi\right\}_{n=0}^{\infty}$ is a frame and one of the involved sequences $\left\{T_{j}^{n} \varphi\right\}_{n=0}^{\infty}$ already is a frame by itself, nothing can be concluded about the range of the remaining operators $T_{j^{\prime}}, j^{\prime} \neq j$.

Due to Proposition 2.3 and Proposition 2.5 we will now devote our attention to linearly independent and redundant frames $\left\{f_{k}\right\}_{k=1}^{\infty}$. Such frames appear at several occasions in the literature. For example, any Gabor frame $\left\{E_{m b} T_{n a} g\right\}_{m, n \in \mathbb{Z}}$ with $a b<1$ is redundant and linearly independent.

We will first prove a general result, showing that if a frame $\left\{f_{k}\right\}_{k=1}^{\infty}$ with finite excess has a representation $\left\{f_{k}\right\}_{k=1}^{\infty}=\left\{T^{n} f_{1}\right\}_{n=0}^{\infty}$, the operator $T$ is forced to be unbounded.

Proposition 2.6 Assume that the frame $\left\{f_{k}\right\}_{k=1}^{\infty}$ is linearly independent, contains a Riesz basis and has finite and strictly positive excess. If $T$ is a linear operator and $\left\{f_{k}\right\}_{k=1}^{\infty}=\left\{T^{n} f_{1}\right\}_{n=0}^{\infty}$, then $T$ is unbounded.

Proof. By assumption there exists $K \in \mathbb{N}$ such that $\left\{f_{k}\right\}_{k=K+1}^{\infty}$ is a Riesz basis for $\widetilde{\mathcal{H}}:=\overline{\operatorname{span}}\left\{f_{k}\right\}_{k=K+1}^{\infty}$ and $\left\{f_{k}\right\}_{k=K}^{\infty}$ is an overcomplete frame for $\widetilde{\mathcal{H}}$ (with excess 1). For $N \in \mathbb{N}$, let $A_{N}$ denote the optimal lower Riesz basis bound for the finite sequence $\left\{f_{k}\right\}_{k=K}^{K+N-1}$, and let $A$ denote a lower Riesz basis bound for $\left\{f_{k}\right\}_{k=K+1}^{\infty}$. Since $\left\{f_{k}\right\}_{k=K}^{\infty}$ is an overcomplete and linearly independent frame it is known that $A_{N} \rightarrow 0$ as $N \rightarrow \infty$; see, e.g., [16] or Proposition 7.1.2 in [6]. Now, for $N \in \mathbb{N}$, take a nonzero sequence $\left\{c_{k}^{(N)}\right\}_{k=K}^{K+N-1}$ such that

$$
\left\|\sum_{k=K}^{K+N-1} c_{k}^{(N)} f_{k}\right\|^{2}=A_{N} \sum_{k=K}^{K+N-1}\left|c_{k}^{(N)}\right|^{2} .
$$


Then

$$
\begin{aligned}
\left\|T \sum_{k=K}^{K+N-1} c_{k}^{(N)} f_{k}\right\|^{2} & =\left\|\sum_{k=K}^{K+N-1} c_{k}^{(N)} f_{k+1}\right\|^{2}=\left\|\sum_{k=K+1}^{K+N} c_{k-1}^{(N)} f_{k}\right\|^{2} \\
& \geq A \sum_{k=K+1}^{K+N}\left|c_{k-1}^{(N)}\right|^{2}=A \sum_{k=K}^{K+N-1}\left|c_{k}^{(N)}\right|^{2} \\
& =\frac{A}{A_{N}}\left\|\sum_{k=K}^{K+N-1} c_{k}^{(N)} f_{k}\right\|^{2} .
\end{aligned}
$$

Since $\frac{A}{A_{N}} \rightarrow \infty$ as $N \rightarrow \infty$, it follows that the operator $T$ is unbounded.

The result in Proposition 2.6 can immediately be extended to certain frames with infinite excess, ordered in a special way. See the comment after Example 2.8 for a more general result.

Corollary 2.7 Assume that the frame $\left\{f_{k}\right\}_{k=1}^{\infty}$ is linearly independent, contains a Riesz basis and has infinite excess. Order the frame elements such that $\left\{f_{1+2 k}\right\}_{k=1}^{\infty}$ is a Riesz basis. Then, if $\left\{f_{k}\right\}_{k=1}^{\infty}=\left\{T^{n} f_{1}\right\}_{n=0}^{\infty}$, the operator $T$ is unbounded.

Proof. Note that $\left\{\left(T^{2}\right)^{n} f_{1}\right\}_{n=0}^{\infty}=\left\{T^{2 n} f_{1}\right\}_{n=0}^{\infty}=\left\{f_{1}\right\} \cup\left\{f_{1+2 k}\right\}_{k=1}^{\infty}$. It now follows from Proposition 2.6 that the operator $T^{2}$ is unbounded. Hence also $T$ is unbounded, as desired.

Example 2.8 Consider a Gabor Riesz basis $\left\{E_{m b} T_{n a} g\right\}_{m, n \in \mathbb{Z}}$ for $L^{2}(\mathbb{R})$. Then, for any $p, q \in \mathbb{N}$ with $p q>1$ the oversampled Gabor system $\left\{E_{m a / p} T_{n b / q} g\right\}_{m, n \in \mathbb{Z}}$ is an overcomplete and linearly independent frame for $L^{2}(\mathbb{R})$. Thus, with the ordering of the system in Corollary 2.7, an operator $T$ such that $\left\{E_{m b} T_{n a} g\right\}_{m, n \in \mathbb{Z}}=$ $\left\{T^{n} \varphi\right\}_{n=0}^{\infty}$ for some $\varphi \in L^{2}(\mathbb{R})$ is necessarily unbounded.

Note that after submission of the current manuscript, a more general result than Corollary 2.7 has been obtained in [8] using more advanced operator theory. Indeed, for a frame containing a Riesz basis and having positive excess, a representation $\left\{f_{k}\right\}_{k=1}^{\infty}=\left\{T^{n} f_{1}\right\}_{n=0}^{\infty}$ is not possible with a bounded operator $T$, regardless of the ordering of the frame elements. The results in [8] also have the consequence that an overcomplete Gabor frame never has a representation of the form $\left\{E_{m b} T_{n a} g\right\}_{m, n \in \mathbb{Z}}=\left\{T^{n} \varphi\right\}_{n=0}^{\infty}$ with a bounded operator $T$.

Returning to the setup of Theorem 2.1, the above results show that in general it is problematic to aim at a reduction of the number of generators in (2.1) to $J=1$. Via an example we will now show that also the question of choosing the operators $T_{j}$ in (2.1) to be independent of $j \in J$ (while keeping the boundedness) has a negative answer in general. 
Example 2.9 Let $\left\{e_{k}\right\}_{k=1}^{\infty}$ denote an orthonormal basis for $\mathcal{H}$, and let

$$
f_{1}:=e_{1}, f_{k}:=e_{k-1}+\frac{1}{k} e_{k}, k \geq 2 .
$$

Then $\left\{f_{k}\right\}_{k=1}^{\infty}$ is a frame [6]; it is linearly independent, and its excess is 1 . Assume that $\left\{f_{k}\right\}_{k=1}^{\infty}=\bigcup_{j=1}^{J}\left\{T^{n} \varphi_{j}\right\}_{n=0}^{\infty}$ for a linear operator $T: \mathcal{H} \rightarrow \mathcal{H}$ and some elements $\varphi_{1}, \ldots, \varphi_{J}$ from $\left\{f_{k}\right\}_{k=1}^{\infty}$. With the purpose of showing that $T$ is unbounded, we will consider the action of $T$ on the elements

$$
g_{N}=\sum_{k=1}^{N} \frac{(-1)^{k-1}}{(k-1) !} f_{k}=\frac{(-1)^{N-1}}{N !} e_{N}
$$

for $N \in \mathbb{N}$. The idea of the following proof is to show that $\left\|T g_{N}\right\|$ is bounded below, independently of $N \in \mathbb{N}$; by (2.2) this implies that $T$ must be unbounded. Technically, the proof is done by analyzing certain subcases separately.

First, since $f_{1}=e_{1}$ we know that $T f_{1} \neq e_{1}$, so $T f_{1}=e_{\ell}+\frac{1}{\ell+1} e_{\ell+1}$ for some $\ell \in \mathbb{N}$. Thus

$$
T g_{N}=\sum_{k=1}^{N} \frac{(-1)^{k-1}}{(k-1) !} T f_{k}=e_{\ell}+\frac{1}{\ell+1} e_{\ell+1}+\sum_{k=2}^{N} \frac{(-1)^{k-1}}{(k-1) !} T f_{k} .
$$

We now split the analysis into some subcases, depending on whether $\ell=1$ or $\ell \geq 2$.

1) If $\ell \geq 2$, then the sum on the right-hand side of (2.3) can only have a component in the direction $e_{\ell}$ if $T f_{k}=e_{\ell-1}+\frac{1}{\ell} e_{\ell}$ for some $k \geq 2$. The smallest component in direction of $e_{\ell}$ appears if this happens for $k=2$, which implies that in any case $\left\|T g_{N}\right\| \geq\left\|\left(1-\frac{1}{2}\right) e_{\ell}\right\|=\frac{1}{2}$. Via (2.2) it follows that $\left\|T e_{N}\right\| \geq \frac{1}{2} N ! \rightarrow \infty$ as $N \rightarrow \infty$. Thus the operator $T$ is unbounded in this case.

2) We now assume that $\ell=1$, in which case (2.3) takes the form

$$
T g_{N}=e_{1}+\frac{1}{2} e_{2}+\sum_{k=2}^{N} \frac{(-1)^{k-1}}{(k-1) !} T f_{k} .
$$

The technical complication is now that it is possible that $T f_{2}=e_{1}$, in which case $T g_{N}$ does not have a component in direction of $e_{1}$ and the above argument breaks down. Again we handle this by splitting into certain subcases:

2a) If $T f_{2}=e_{1},(2.4)$ takes the form

$$
T g_{N}=\frac{1}{2} e_{2}+\sum_{k=3}^{N} \frac{(-1)^{k-1}}{(k-1) !} T f_{k} .
$$


Hence we will now focus on the possible components of $T f_{k}$ in direction of $e_{2}$ for $k \geq 3$. Since $T f_{1}=e_{1}+\frac{1}{2} e_{2}$ by assumption, the only possibility for such a component is that $T f_{k}=e_{2}+\frac{1}{3} e_{3}$ for some $k \geq 3$. In this case (2.5) implies that the component of $T g_{N}$ in direction of $e_{2}$ is $\frac{1}{2} e_{2}+\frac{(-1)^{k-1}}{(k-1) !} e_{2}$ hence $\left\|T g_{N}\right\| \geq \frac{1}{2}-\frac{1}{6}=\frac{1}{3}$. Via the same argument as in 1 ) we conclude that $T$ is unbounded.

2b) Finally, if $T f_{2} \neq e_{1}$, (2.4) implies that the component of $T g_{N}$ in direction of $e_{1}$ is at least $e_{1}-\frac{1}{6} e_{1}=\frac{5}{6} e_{1}$; thus $\left\|T g_{N}\right\| \geq \frac{5}{6}$, and $T$ is also unbounded in this case.

It is natural to ask whether the condition of linear independence of a frame in Proposition 2.3 implies that the frame is norm-bounded below (the condition in Theorem 2.1). For frames with finite excess the answer is yes; however, the following example shows that in any separable Hilbert space we can construct linearly independent frames with infinite excess, which are not norm-bounded below.

Example 2.10 Let $\left\{e_{k}\right\}_{k=1}^{\infty}$ denote an orthonormal basis for $\mathcal{H}$. We will describe a recursive procedure which yields a sequence $\left\{\varphi_{k}\right\}_{k=1}^{\infty}$ such that $\left\{f_{k}\right\}_{k=1}^{\infty}:=$ $\left\{e_{k}\right\}_{k=1}^{\infty} \cup\left\{\varphi_{k}\right\}_{k=1}^{\infty}$ is a linearly independent frame which is not norm-bounded below. In the first step, choose infinite and disjoint index sets $I_{1}$ and $J_{1}$ such that $\mathbb{N}=I_{1} \cup J_{1}$. Letting $\varphi_{1}:=\sum_{\ell \in I_{1}} \frac{1}{\sqrt{2}^{\ell}} e_{\ell}$, the set $\left\{e_{k}\right\}_{k \in I_{1}} \cup\left\{\varphi_{1}\right\}$ is a linearly independent frame sequence with excess one. We now continue this procedure in a recursive way starting with the orthonormal sequence $\left\{e_{k}\right\}_{k \in J_{1}}$. To be more precise, in the $n$th step we choose infinite and disjoint sets $I_{n}$ and $J_{n}$ such that $J_{n-1}=I_{n} \cup J_{n}$. Defining $\varphi_{n}:=n^{-1} \sum_{\ell \in I_{n}} \frac{1}{\sqrt{2}^{\ell}} e_{\ell}$, we then see that $\left\{e_{k}\right\}_{k \in I_{n}} \cup\left\{\varphi_{n}\right\}$ is a linearly independent frame sequence with excess one. By construction the spaces spanned by these frame sequences form an orthogonal decomposition of $\mathcal{H}$. It follows that the sequence $\left\{e_{k}\right\}_{k=1}^{\infty} \cup\left\{\varphi_{k}\right\}_{k=1}^{\infty}=\bigcup_{n=1}^{\infty}\left(\left\{e_{k}\right\}_{k \in I_{n}} \cup\left\{\varphi_{n}\right\}\right)$ is a linearly independent frame for $\mathcal{H}$; and since $\left\|\varphi_{n}\right\| \rightarrow 0$ as $n \rightarrow \infty$, it is not norm-bounded below.

Note that for each $\epsilon \in] 0,1\left[\right.$ the frame $\left\{f_{k}\right\}_{k=1}^{\infty}$ consists of

- Infinitely many elements with norm 1;

- Infinitely many elements with norm at most $\epsilon$;

- Finitely many elements with norm between $\epsilon$ and 1 .

Reordering the frame as $\left\{f_{k}\right\}_{k=1}^{\infty}$ and defining the operator $T: \operatorname{span}\left\{f_{k}\right\}_{k=1}^{\infty} \rightarrow$ $\mathcal{H}$ by $T f_{k}=f_{k+1}$, this implies that for any $\left.\epsilon \in\right] 0,1[$ there exists some $j \in \mathbb{N}$ such that $\left\|f_{j}\right\| \leq \epsilon$ and $\left\|f_{j+1}\right\|=1$. Thus $\left\|T \frac{f_{j}}{\left\|f_{j}\right\|}\right\|=\frac{\left\|f_{j+1}\right\|}{\left\|f_{j}\right\|} \geq \frac{1}{\epsilon}$, i.e., $T$ is unbounded. 


\section{Proof of Theorem 2.1}

We will need some preparation before we prove Theorem 2.1.

Lemma 3.1 Fix $N \in \mathbb{N}$. Assume that $\left\{f_{k}\right\}_{k=1}^{N} \cup\left\{g_{k}\right\}_{k=1}^{\infty}$ is a Bessel sequence in $\mathcal{H}$ and let $P$ denote the orthogonal projection onto span $\left\{f_{k}\right\}_{k=1}^{N}$. Then, for any $\epsilon>0$ there is some $j \in \mathbb{N}$ such that $\left\|P g_{j}\right\| \leq \epsilon$.

Proof. The proof is by contradiction, so assume that $\left\|P g_{k}\right\|>\epsilon>0$ for all $k \in \mathbb{N}$. Since $\left\{g_{k}\right\}_{k=1}^{\infty}$ is a Bessel sequence it is norm-bounded above, and therefore also $\left\{P g_{k}\right\}_{k=1}^{\infty}$ is norm-bounded. Thus, for some $R>0$ it belongs to the ball $B(0, R):=\left\{f \in \operatorname{span}\left\{f_{k}\right\}_{k=1}^{N} \mid\|f\| \leq R\right\}$ in the finite-dimensional space $\operatorname{span}\left\{f_{k}\right\}_{k=1}^{N}$. By compactness of the ball, the sequence thus has a convergent subsequence, say $P g_{k_{\ell}} \rightarrow h$ for $\ell \rightarrow \infty$. Clearly $\|h\| \geq \epsilon$ and $h \in \operatorname{span}\left\{f_{k}\right\}_{k=1}^{N}$. However, since

$$
\sum_{k=1}^{\infty}\left|\left\langle h, g_{k}\right\rangle\right|^{2}=\sum_{k=1}^{\infty}\left|\left\langle P h, g_{k}\right\rangle\right|^{2}=\sum_{k=1}^{\infty}\left|\left\langle h, P g_{k}\right\rangle\right|^{2} \geq \sum_{\ell=1}^{\infty}\left|\left\langle h, P g_{k_{\ell}}\right\rangle\right|^{2}=\infty
$$

this contradicts $\left\{g_{k}\right\}_{k=1}^{\infty}$ being a Bessel sequence. Thus we conclude that there indeed exists $j \in \mathbb{N}$ such that $\left\|P g_{j}\right\| \leq \epsilon$.

Lemma 3.2 Let $N \in \mathbb{N}$ be fixed. Assume that $\left\{f_{k}\right\}_{k=1}^{N}$ and $\left\{g_{k}\right\}_{k=1}^{\infty}$ are Riesz sequences, let $C:=\inf _{j, k \in \mathbb{N}}\left\{\left\|f_{k}\right\|,\left\|g_{j}\right\|\right\}$, and let $A<C$ denote a common lower Riesz bound for $\left\{f_{k}\right\}_{k=1}^{N}$ and $\left\{g_{k}\right\}_{k=1}^{\infty}$. Given any $\left.\delta \in\right] 0, A[$, choose $j \in \mathbb{N}$ as in Lemma 3.1 with $\epsilon<\min \{C-A, \sqrt{A}-\sqrt{A-\delta}\}$. Then $\left\{f_{k}\right\}_{k=1}^{N} \cup\left\{g_{k}\right\}_{k=1}^{\infty}$ is a Riesz sequence with lower bound $A-\delta$.

Proof. Let $P$ denote the orthogonal projection onto $\operatorname{span}\left\{f_{k}\right\}_{k=1}^{N}$, and choose $j \in \mathbb{N}$ such that $\left\|P g_{j}\right\| \leq \epsilon$. Then $\left\|(I-P) g_{j}\right\| \geq C-\epsilon>A$. For notational convenience and just for this step of the proof, let us put $f_{N+1}:=(I-P) g_{j}$. Noting that $f_{N+1}$ is perpendicular to all $f_{k}, k=1, \ldots, N$, for any sequence of scalar coefficients $c_{1}, \ldots, c_{N+1}$ we have

$$
\begin{aligned}
\left\|\sum_{k=1}^{N+1} c_{k} f_{k}\right\|^{2} & =\left\|\sum_{k=1}^{N} c_{k} f_{k}\right\|^{2}+\left|c_{N+1}\right|^{2}\left\|f_{N+1}\right\|^{2} \\
& \geq A \sum_{k=1}^{N}\left|c_{k}\right|^{2}+A\left|c_{N+1}\right|^{2}=A \sum_{k=1}^{N+1}\left|c_{k}\right|^{2}
\end{aligned}
$$

Thus $\left\{f_{k}\right\}_{k=1}^{N+1}$ is a Riesz sequence with lower bound $A$. Now, letting $\left\{h_{k}\right\}_{k=1}^{N+1}:=$ $\left\{f_{1}, \ldots, f_{N}, g_{j}\right\}$, we see that for any scalar coefficients $c_{1}, \ldots, c_{N+1}$,

$$
\left\|\sum_{k=1}^{N+1} c_{k}\left(f_{k}-h_{k}\right)\right\|^{2}=\left|c_{N+1}\right|^{2}\left\|f_{N+1}-h_{N+1}\right\|^{2}=\left|c_{N+1}\right|^{2}\left\|P g_{j}\right\|^{2} \leq \epsilon^{2} \sum_{k=1}^{N+1}\left|c_{k}\right|^{2} .
$$


By assumption, $\epsilon<\sqrt{A}-\sqrt{A-\delta}<\sqrt{A}$; thus, classical perturbation results (see, e.g., [6], Theorem 22.3.2) imply that $\left\{h_{k}\right\}_{k=1}^{N+1}$ is a Riesz sequence with lower bound

$$
A\left(1-\frac{\epsilon}{\sqrt{A}}\right)^{2} \geq A\left(1-\frac{\sqrt{A}-\sqrt{A-\delta}}{\sqrt{A}}\right)^{2}=A-\delta,
$$

as desired.

\section{Proof of Theorem 2.1:}

The Feichtinger conjecture (which has been confirmed in [18] in 2013 based on the equivalent formulations in [5] and therefore now is a theorem) implies that the frame can be partitioned into a finite union of Riesz sequences,

$$
\left\{f_{k}\right\}_{k=1}^{\infty}=\bigcup_{j=1}^{J}\left\{f_{k}^{(j)}\right\}_{k \in I_{j}}
$$

for certain index sets $I_{j}, j=1, \ldots, J$. We will first argue that all of these Riesz sequences can be chosen to contain infinitely many elements. Let $A$ denote a common lower frame bound for each of the Riesz sequences $\left\{f_{k}^{(j)}\right\}_{k \in I_{j}}, j=$ $1, \ldots, J$, chosen such that $A<\inf _{k \in \mathbb{N}}\left\|f_{k}\right\|$. At least one of these Riesz sequences, say, $\left\{f_{k}^{(\alpha)}\right\}_{k \in I_{\alpha}}$ will contain infinitely many elements. Now assume that another one of the Riesz sequences, say, $\left\{f_{k}^{(\beta)}\right\}_{k \in I_{\beta}}$, only contains finitely many elements. Now, applying Lemma 3.2 with $\delta:=A 2^{-\ell_{1}}$ for some $\ell_{1} \geq 2$ we can find an element $f_{k_{1}}^{(\alpha)}, k_{1} \in I_{\alpha}$, such that $\left\{f_{k}^{(\beta)}\right\}_{k \in I_{\beta}} \cup\left\{f_{k_{1}}^{(\alpha)}\right\}$ is a Riesz sequence with lower bound $A-A 2^{-\ell_{1}}$. We will now apply this procedure in an inductive way, in the next step starting with the Riesz sequence $\left\{f_{k}^{(\beta)}\right\}_{k \in I_{\beta}} \cup\left\{f_{k_{1}}^{(\alpha)}\right\}$ and choosing $\delta=A 2^{-\ell_{2}}$ for some $\ell_{2}>\ell_{1}+1$. Since the Riesz sequence property (see (3.29) in [6]) holds if it just holds on arbitrary finite sequences, this procedure will yield an infinite Riesz sequence

$$
\left\{f_{k}^{(\beta)}\right\}_{k \in I_{\beta}} \cup\left\{f_{k_{1}}^{(\alpha)}\right\} \cup\left\{f_{k_{2}}^{(\alpha)}\right\} \cup \cdots,
$$

with lower bound $A-A\left(2^{-\ell_{1}}+2^{-\ell_{2}}+\ldots\right) \geq A\left(1-2^{-\ell_{1}+1}\right)$. Performing this procedure on each of the finite Riesz sequences in the decomposition (3.1) now proves that all of the Riesz sequences can be chosen to be infinite. Finally, since the described procedure involves a "gap" between the removed elements (due to the choice $\ell_{2}>\ell_{1}+1$ ) the remaining part of $\left\{f_{k}^{(\alpha)}\right\}_{k \in I_{\alpha}}$ is still an infinite sequence. The desired result is thus a consequence of Proposition 2.5.

Remark: The proof of Theorem 2.1 shows that by choosing the integer $\ell_{1}$ sufficiently large, we can obtain that the Riesz sequences in (2.1) have a lower 
Riesz bound that is arbitrarily close to the considered joint lower Riesz bound for the sequences in (3.1).

Acknowledgment The authors would like to thank the anonymous reviewers for their comments, which improved the presentation of the results.

\section{References}

[1] Aldroubi, A., Cabrelli, C., Çakmak, A. F., Molter, U., and Petrosyan, A.: Iterative actions of normal operators. J. Funct. Anal. 272 (2017), $1121-1146$.

[2] Aldroubi, A., Cabrelli, C., Molter, U., and Tang, S.: Dynamical sampling. Appl. Comput. Harmon. Anal. 42 (2017), 378-401.

[3] Aldroubi, A., and Petrosyan, A.: Dynamical sampling and systems from iterative actions of operators. In: Pesenson I., Le Gia Q., Mayeli A., Mhaskar H., Zhou DX. (eds), Frames and other bases in abstract and function spaces. Applied and Numerical Harmonic Analysis. Birkhäuser, Boston, 2017.

[4] Bownik, M., and Speegle, D.: Linear independence of Parseval wavelets. Illinois J. Math. 54 (2010), 771-785.

[5] Casazza, P. G., Fickus, M., Tremain, J. C., and Weber, E.: The KadisonSinger problem in mathematics and engineering - a detailed account. Contemp. Math. 414 (2006), 297-356.

[6] Christensen, O.: An introduction to frames and Riesz bases. Second expanded edition. Birkhäuser, Boston, 2016.

[7] Christensen, O., Deng, B., and Heil, C.: Density of Gabor frames. Appl. Comp. Harm. Anal. 7 (1999), 292-304.

[8] Christensen, O., Hasannasab, M., and Philipp, F.: Frame Properties of Operator Orbits. Preprint, 2018.

[9] Christensen, O. and Lindner, A.: Lower bounds for finite Gabor and wavelet systems. Appr. Theory and its Appl. 17 no.1 (2001), 18-29.

[10] Daubechies, I.: Ten lectures on wavelets. SIAM, Philadelphia, 1992.

[11] Fernandez-Morales, H., Garcia, A. G., Hernandez-Medina, M. A., and Munos-Bouzo, M. J.: Generalized sampling: from shift-invariant to Uinvariant spaces. Anal. Appl. 13 (2015), no. 3, 303-329. 
[12] Garcia, A. G. and Munos-Bouzo, M. J.:Sampling-related frames in finite U-invariant subspaces. Appl. Comput. Harmon. Anal. 39 (2015), no. 1, 173-184.

[13] Gröchenig, K.: Foundations of time-frequency analysis. Birkhäuser, Boston, 2000.

[14] Heil, C.: Linear independence of finite Gabor systems. In "Harmonic analysis and applications", 171-206, Appl. Numer. Harmon. Anal., Birkhäuser Boston, 2006.

[15] Heil, C., Ramanathan, J. and Topiwala, P.: Linear independence of timefrequency translates. Proc. Amer. Math. Soc. 124 (1996), 2787-2795.

[16] Kim, H.O. and Lim, J.K.: New characterizations of Riesz bases. Appl. Comp. Harm. Anal. 4 (1997), 222-229.

[17] Linnell, P.: Von Neumann algebras and linear independence of translates. Proc. Amer. Math. Soc. 127 no. 11 (1999), 3269-3277.

[18] Marcus, A. W., Spielman, D. A., and Srivastava, N.: Interlacing families II: Mixed characteristic polynomials and the Kadison-Singer problem. Ann. of Math. (2) 182 no. 1, (2015), 327-350.

[19] Philipp, F.: Bessel orbits of normal operators., J. Math. Anal. Appl. 448 (2017), 767-785.

Ole Christensen

Technical University of Denmark

DTU Compute

Building 303, 2800 Lyngby

Denmark

Email: ochr@dtu.dk
Marzieh Hasannasab

Technical University of Denmark DTU Compute

Building 303, 2800 Lyngby

Denmark

mhas@dtu.dk 